\title{
Prime Focus Spectrograph (PFS) for the Subaru Telescope: Overview, recent progress, and future perspectives
}

Naoyuki Tamura $^{\mathrm{a}}$, Naruhisa Takato ${ }^{\mathrm{b}}$, Atsushi Shimono ${ }^{\mathrm{a}}$, Yuki Moritani ${ }^{\mathrm{a}}$, Kiyoto Yabe ${ }^{\mathrm{a}}$, Yuki Ishizuka $^{a}$, Akitoshi Uedac ${ }^{\text {, Yukiko Kamata }}{ }^{\text {, Hrand Aghazarian }}{ }^{\text {, }}$, Stéphane Arnouts ${ }^{\mathrm{e}}$, Gabriel

Barban $^{\text {f }}$, Robert H. Barkhouserg ${ }^{\text {, Renato C. Borges }}{ }^{\mathrm{e}}$, David F. Braun ${ }^{\mathrm{d}}$, Michael A. Carr ${ }^{\mathrm{h}}$,

Pierre-Yves Chabaud ${ }^{e}$, Yin-Chang Chang ${ }^{i}, H_{\text {sin-Yo Chen }}{ }^{i}$, Masashi Chiba ${ }^{j}$, Richard C. Y.

Chou $^{\mathrm{i}}$, You-Hua Chu ${ }^{\mathrm{i}}$, Judith G. Cohen ${ }^{\mathrm{k}}$, Rodrigo P. de Almeida ${ }^{\mathrm{f}}$, Antonio C. de Oliveira ${ }^{\mathrm{f}}$, Ligia S. de Oliveira ${ }^{\mathrm{f}}$, Richard G. Dekany ${ }^{\mathrm{k}}$, Kjetil Dohlen ${ }^{\mathrm{e}}$, Jesulino B. dos Santos ${ }^{\mathrm{f}}$, Leandro H. dos Santos $^{\mathrm{f}}$, Richard S. Ellis ${ }^{\mathrm{l}, \mathrm{m}}$, Maximilian Fabricius ${ }^{\mathrm{b}}$, Didier Ferrand ${ }^{\mathrm{e}}$, Décio Ferreira ${ }^{\mathrm{f}}$, Mirek Golebiowski $^{\mathrm{g}}$, Jenny E. Greene ${ }^{\mathrm{h}}$, Johannes Gross ${ }^{\mathrm{d}}$, James E. Gunn ${ }^{\mathrm{h}}$, Randolph Hammond ${ }^{\mathrm{g}}$,

Albert Hardings, Murdock Hart ${ }^{\mathrm{g}}$, Timothy M. Heckman ${ }^{\mathrm{g}}$, Christopher M. Hiratan ${ }^{\mathrm{n}}$, Paul Ho ${ }^{\mathrm{i}}$,

Stephen C. Hope ${ }^{g}$, Larry Hovland ${ }^{\mathrm{d}}$, Shu-Fu Hsu ${ }^{\mathrm{i}}$, Yen-Shan Hu${ }^{\mathrm{i}}$, Ping-Jie Huang ${ }^{\mathrm{i}}$, Marc

Jaquet $^{\mathrm{e}}$, Yipeng Jing ${ }^{\mathrm{o}}$, Jennifer Karr ${ }^{\mathrm{i}}$, Masahiko Kimura ${ }^{\mathrm{i}}$, Matthew E. King ${ }^{\mathrm{d}}$, Eiichiro

Komatsu $^{\mathrm{a}, \mathrm{p}}$, Vincent Le Brun ${ }^{\mathrm{e}}$, Olivier Le Févre ${ }^{\mathrm{e}}$, Arnaud Le Fur ${ }^{\mathrm{e}}$, David Le Mignant ${ }^{\mathrm{e}}$, Hung-Hsu Ling ${ }^{\mathrm{i}}$, Craig P. Loomis ${ }^{\mathrm{h}}$, Robert H. Lupton ${ }^{\mathrm{h}}$, Fabrice Madec ${ }^{\mathrm{e}}$, Peter Mao ${ }^{\mathrm{k}}$, Lucas S.

Marrara $^{\mathrm{f}}$, Claudia Mendes de Oliveira ${ }^{\mathrm{q}}$, Yosuke Minowa ${ }^{\mathrm{b}}$, Chaz N. Morantz ${ }^{\mathrm{d}}$, Hitoshi

Murayama $^{\mathrm{a}, \mathrm{r}, \mathrm{s}}$, Graham J. Murray ${ }^{\mathrm{t}}$, Youichi Ohyama ${ }^{\mathrm{i}}$, Joseph Orndorffr ${ }^{\mathrm{g}}$, Sandrine Pascal ${ }^{\mathrm{e}}$,

Jefferson M. Pereira ${ }^{\mathrm{f}}$, Daniel J. Reiley ${ }^{\mathrm{k}}$, Martin Reinecke ${ }^{\mathrm{p}}$, Andreas Ritter ${ }^{\mathrm{h}}$, Mitsuko Roberts ${ }^{\mathrm{k}}$,

Mark A. Schwochert ${ }^{d}$, Michael D. Seiffert ${ }^{d}$, Stephen A. Smee ${ }^{g}$, Laerte Sodre Jr. ${ }^{\mathrm{q}}$, David N.

Spergel $^{\text {h }}$, Aaron J. Steinkraus ${ }^{\mathrm{d}}$, Michael A. Strauss ${ }^{\mathrm{h}}$, Christian Surace ${ }^{\mathrm{e}}$, Yasushi Suto ${ }^{\mathrm{u}, \mathrm{v}}$, Nao

Suzuki $^{\mathrm{a}}$, John Swinbank ${ }^{\mathrm{h}}$, Philip J. Tait ${ }^{\mathrm{b}}$, Masahiro Takada ${ }^{\mathrm{a}}$, Tomonori Tamura ${ }^{\mathrm{b}}$, Yoko

Tanaka $^{\mathrm{b}}$, Laurence Tresse ${ }^{\mathrm{e}, \mathrm{w}}$, Orlando Verducci Jr. ${ }^{\mathrm{f}}$, Didier Vibert ${ }^{\mathrm{e}}$, Clement Vidal ${ }^{\mathrm{e}}$, Shiang-Yu

Wang $^{\mathrm{i}}$, Chih-Yi Wen ${ }^{\mathrm{i}}$, Chi-Hung Yan ${ }^{\mathrm{i}}$, and Naoki Yasuda ${ }^{\mathrm{a}}$

${ }^{a}$ Kavli Institute for the Physics and Mathematics of the Universe (WPI), The University of

Tokyo Institutes for Advanced Study, The University of Tokyo, Kashiwa, Chiba 277-8583, Japan

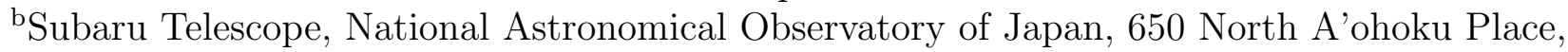
Hilo, HI 96720, USA

${ }^{\mathrm{c}}$ National Astronomical Observatory of Japan, 2-21-1 Osawa, Mitaka, Tokyo 181-8588, Japan

d Jet Propulsion Laboratory, 4800 Oak Grove Dr., Pasadena, CA 91109, USA

eAix Marseille Université, CNRS, LAM (Laboratoire d'Astrophysique de Marseille) UMR

7326, 13388, Marseille, France

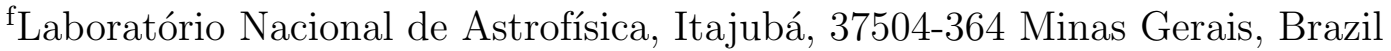

g.Johns Hopkins University, Department of Physics and Astronomy, 3701 San Martin Drive,

Baltimore, MD 21218, USA

${ }^{\text {h}}$ Princeton University, Department of Astrophysical Sciences, Princeton, NJ 08544, USA

${ }^{i}$ Academia Sinica, Institute of Astronomy and Astrophysics, P. O. Box 23-141, Taipei, Taiwan

jTohoku University, Astronomical Institute, Sendai, Miyagi 980-8578, Japan

${ }^{\mathrm{k}}$ California Institute of Technology, 1200 E California Blvd, Pasadena, CA 91125, USA

${ }^{1}$ European Southern Observatory (ESO), Karl-Schwarzschild-Strasse 2, 85748 Garching,

Germany

${ }^{m}$ Department of Physics and Astronomy, University College London, Gower Street, London, WC1E 6BT, UK

Ground-based and Airborne Instrumentation for Astronomy VI, edited by Christopher J. Evans, Luc Simard, Hideki Takami Proc. of SPIE Vol. 9908, 99081M · C 2016 SPIE · CCC code: 0277-786X/16/\$18 · doi: 10.1117/12.2232103 
${ }^{\mathrm{n}}$ Center for Cosmology and Astroparticle Physics, The Ohio State University, 191 West

Woodruff Lane, Columbus, Ohio 43210, USA

${ }^{\circ}$ Center for Astronomy and Astrophysics, Department of Physics and Astronomy, Shanghai

Jiao Tong University, Shanghai 200240, China

pMax-Planck-Institut für Astrophysik, Karl-Schwarzschild Str. 1, D-85741 Garching, Germany

qDepartamento de Astronomia, Instituto de Astronomia, Geofísica e Ciências Atmosféricas, Universidade de São Paulo, Rua do Matão 1226, Cidade Universitária, 05508-090 São Paulo, Brazil

${ }^{\mathrm{r}}$ University of California, Berkeley, CA 94720, USA

'Lawrence Berkeley National Laboratory, MS 50A-5104, Berkeley, CA 94720, USA

${ }^{\mathrm{t}}$ Centre for Advanced Instrumentation, Durham University, South Road, Durham, DH1 3LE, UK

unepartment of Physics, The University of Tokyo, Tokyo 113-0033, Japan

${ }^{v}$ Research Center for the Early Universe, School of Science, The University of Tokyo, Tokyo 113-0033, Japan

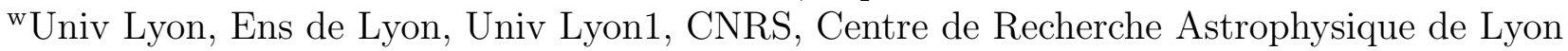
UMR5574, F-69007, Lyon, France

\begin{abstract}
PFS (Prime Focus Spectrograph), a next generation facility instrument on the 8.2-meter Subaru Telescope, is a very wide-field, massively multiplexed, optical and near-infrared spectrograph. Exploiting the Subaru prime focus, 2394 reconfigurable fibers will be distributed over the $1.3 \mathrm{deg}$ field of view. The spectrograph has been designed with 3 arms of blue, red, and near-infrared cameras to simultaneously observe spectra from $380 \mathrm{~nm}$ to $1260 \mathrm{~nm}$ in one exposure at a resolution of $\sim 1.6-2.7 \AA$. An international collaboration is developing this instrument under the initiative of Kavli IPMU. The project is now going into the construction phase aiming at undertaking system integration in 2017-2018 and subsequently carrying out engineering operations in 2018-2019. This article gives an overview of the instrument, current project status and future paths forward.
\end{abstract}

Keywords: Subaru Telescope, future instrument, wide-field instrument, multi-object spectroscopy, optical and near-infrared spectroscopy, optical spectroscopy, near-infrared spectroscopy, international collaboration, optical fibers

\title{
1. INTRODUCTION
}

The wide-field capability at the prime focus is clearly one of the key advantages of the $8.2 \mathrm{~m}$ Subaru Telescope, and a few instruments are exploiting this to deliver valuable scientific data. Suprime-Cam (Subaru Prime Focus Camera $)^{1}$ is a wide-field imager with a mosaic of ten $2 \mathrm{~K} \times 4 \mathrm{~K}$ CCDs covering a field of $34^{\prime} \times 27^{\prime}$. Its broadband and narrow-band deep and wide imaging data have been powerful for a number of discoveries and detailed characterization of various astronomical objects over a wide range of redshifts. FMOS (Fiber Multi-Object Spectrograph $)^{2,3}$ is a wide-field, fiber-fed multi-object spectrograph, where the 400 fibers on the half-degree field are reconfigurable with an Echidna-style fiber positioner system. ${ }^{4}$ The spectra cover the near-infrared regime from $900 \mathrm{~nm}$ to $1800 \mathrm{~nm}$. This allows highly efficient observation of the rest-frame optical spectral features for objects at redshifts beyond one (e.g. ${ }^{5}$ ) and absorption bands in the infrared continuum of cool, low-mass stars (e.g. ${ }^{6}$ ). Recently, the Subaru Telescope observatory has started accepting a large program in the framework called "Subaru Strategic Program" (SSP) that can span up to $\sim 300$ nights over $\sim 5$ years. A cosmology survey program called "FastSound" ${ }^{7}$ (PI: T. Totani), one of the few SSP programs, was successfully completed with

Further author information: (Send correspondence to Naoyuki Tamura.)

E-mail: naoyuki.tamura@ipmu.jp, Telephone: +81 (0)4 71366531 

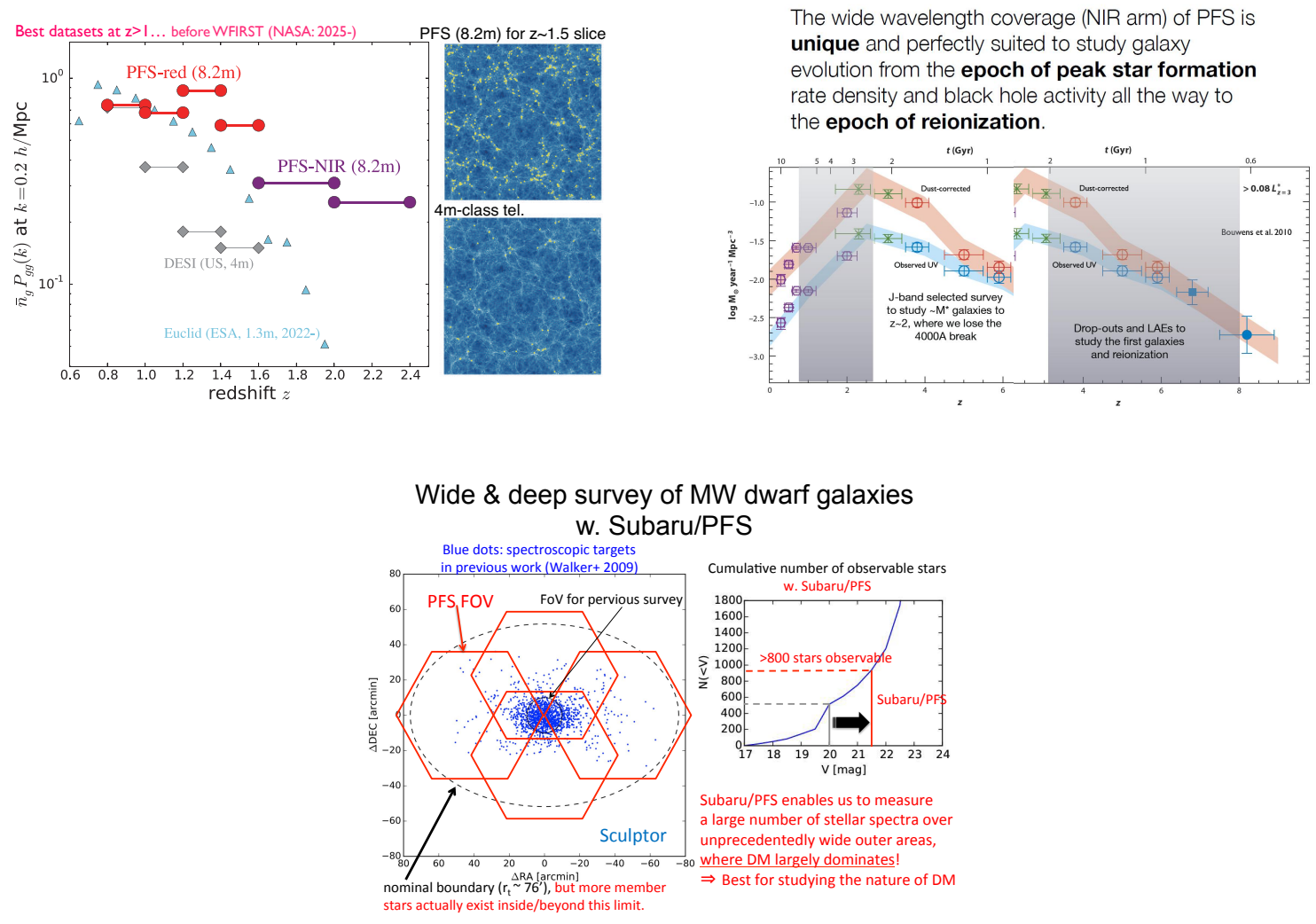

Figure 1. A few representations of the PFS strengths. Top left: the product of the mean galaxy space density and the galaxy clustering amplitude is plotted as a function of redshift and the case for the PFS cosmology program is compared with WFIRST and DESI. ${ }^{10,11}$ Top right: the cosmic star formation history ${ }^{12}$ is illustrated with a wide range of redshifts that can be accessed in the PFS galaxy \& AGN evolution survey exploiting the wide wavelength coverage. Bottom: a PFS pointing plan around the Sculptor dwarf galaxy ${ }^{13,14}$ as a part of the Galactic archaeology program is summarized, showing high efficiency thanks to the wide field and high multiplicity.

FMOS to reveal a 3D map of $\sim 3000$ galaxies around $z \sim 1.4$ and a significant detection of Redshift Space Distortions (RSD). ${ }^{8}$

Now new instrumentation projects are underway to upgrade the Subaru prime focus and push the cutting edge science further forward, taking the full advantage of the unique wide field of view. Hyper Suprime-Cam (HSC), ${ }^{9}$ the successor of Suprime-Cam, is a very wide-field imager with a 1.5-degree diameter field of view "paved" by $1162 \mathrm{~K} \times 4 \mathrm{~K}$ CCDs. It has been in science operation since 2014 and a 5 -year, 300-night SSP survey program is ongoing. PFS (Prime Focus Spectrograph), as described in this article, is a very wide-field, massively multiplexed, optical and near-infrared (NIR) spectrometer. The focal plane will be equipped with 2394 reconfigurable fibers distributed in the 1.3-degree wide hexagonal field of view. The spectrograph has been designed to cover a wide range of wavelengths simultaneously from $380 \mathrm{~nm}$ to $1260 \mathrm{~nm}$ in one exposure. The PFS and HSC instrumentation projects are under the umbrella of the Subaru Measurement of Images and Redshifts (SuMIRe) project (PI: H. Murayama) aiming to conduct deep and wide sky surveys exploiting the unique capability of the Subaru Telescope. It should be emphasized that HSC and PFS enable deep imaging and spectroscopic surveys of the same region of sky using the same $8.2 \mathrm{~m}$ telescope, allowing one to have good understandings of various systematics in the data.

Envisioning a large survey in the SSP framework, the PFS science team has built a preliminary survey plan and has developed top-level requirements for the instrument. ${ }^{15}$ The goal is to address key questions in three 


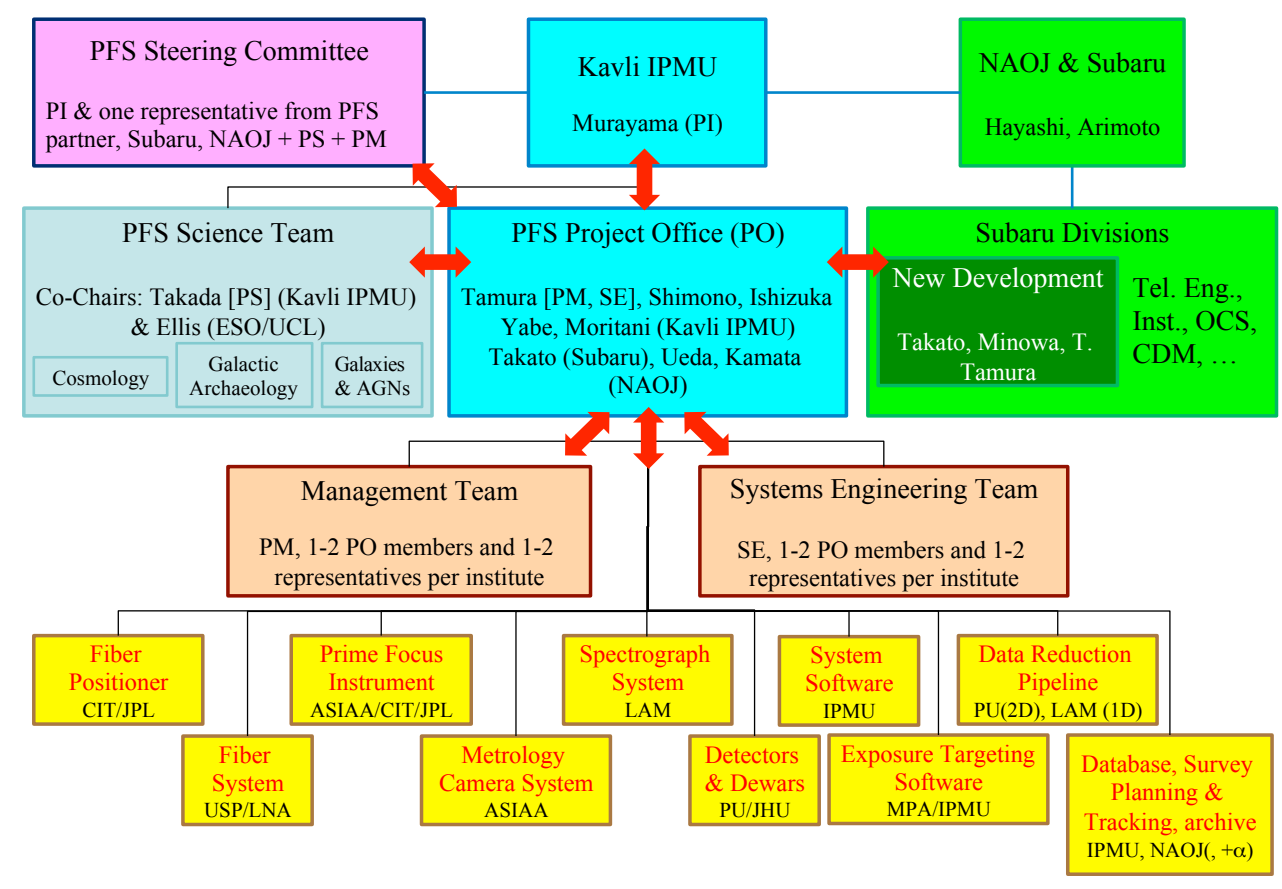

Figure 2. A chart showing the PFS organization and the assignments of instrument subsystems and subcomponents to the collaboration.

main fields: cosmology, galaxy \& AGN evolution, and Galactic archaeology, and from the joint implications, to understand the dark sector of the universe. The team has been continuously refining the plan as the instrument characteristics and technical constraints on the survey observation process are better understood. The combination of the wide field, high multiplicity, and high number density of the fibers on the focal plane offers an opportunity of designing a unique survey on these three core science cases envisioned in the PFS SSP survey (Fig. 1).

The development of this instrument has been undertaken by an international collaboration at the initiative of Kavli IPMU, with work packages for subsystem and subcomponent development assigned to various collaborating institutions (Fig. 2). The project is now in the phase of construction, integration and test aiming to start science operations from mid-late 2019. In parallel, detailed modeling of the instrument and output spectral images are ongoing in order to characterize the instrument on-sky capabilities and accordingly finalize the SSP survey design. This way, a PFS SSP program for follow-up spectroscopy can start in a timely manner subsequently after the HSC SSP survey. In what follows, the instrument basics are described in $\S 2$, and an overview of the instrument and survey operation concept is given in $\S 3$. Then updates of a few major aspects of the project are summarized in $\S 4$, and this article is summarized and a timeline for the future developments is given in $\S 5$.

\section{THE INSTRUMENT}

The PFS instrument is composed of four subsystems, whose distribution on the telescope is illustrated in Fig. 3: The lights from astronomical objects and sky are fed to the fibers configured at the Subaru prime focus, are then transmitted via the fiber cable to the spectrograph system in the telescope enclosure building, and the spectral images of them are delivered on the spectrograph detectors. We here give an overview of these subsystems. The instrumental software system and survey operations are described in $\S 3$. 


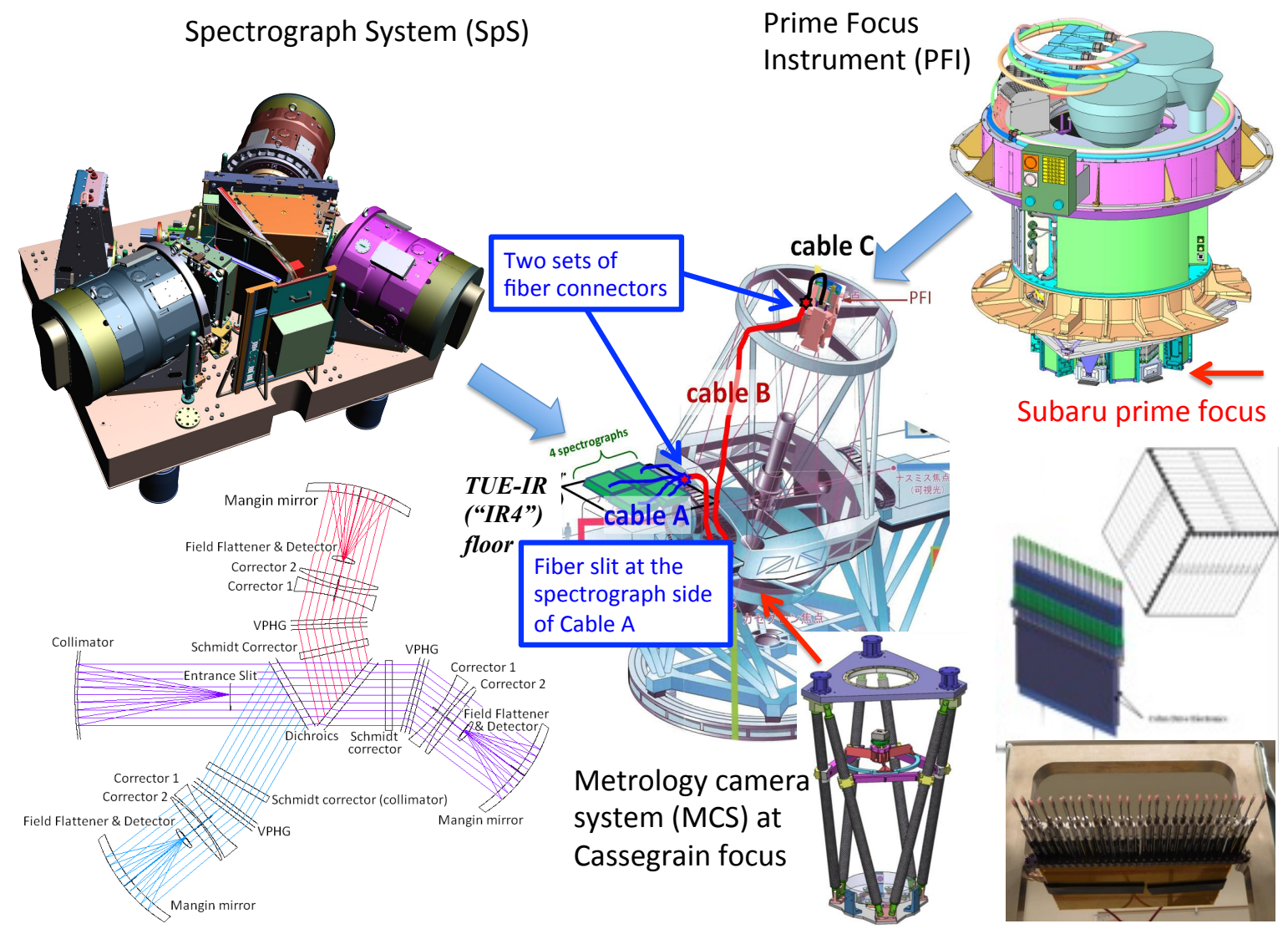

Figure 3. A schematic view of the configuration of PFS subsystems. An overall sketch of the Subaru Telescope is presented in the middle with the PFS fiber cable routed from the prime focus to the spectrograph system. On the right, a solid model of PFI (top), a schematic view of the focal plane (middle), and a photo of the Cobra engineering model fiber positioners module are presented. On the left, a solid model of one spectrograph module (top) and a ray-trace view of it (bottom) are shown. 
At the Subaru prime focus, HSC has already been in science operation with the wide field of view and the reasonably flat focal plane provided by the new Wide-Field Corrector lens system (WFC). The WFC will be used for PFS as well. Mechanically, the new prime focus housing unit "POpt2" is integrated with WFC and accommodates the HSC instrument inside. When PFS is in operation, the HSC instrument will be taken out and our Prime Focus Instrument (PFI) will be installed in POpt2.

PFI has been developed by the collaboration of $\mathrm{CIT}^{*} \&$ NASA $\mathrm{JPL}^{\dagger}, \mathrm{LNA}^{\ddagger}$, and ASIAA ${ }^{\S}$, accommodating key subcomponents such as the fiber positioner system, science \& fiducial fiber system, Acquisition \& Guide (AG) cameras, and calibration system. The fiber positioner system consists of 42 modules each of which accommodates 57 "Cobra" rotary actuators populated with science fibers. The tip of each science fiber is equipped with a planoconcave microlens to increase the focal ratio of the input beam to the fiber to $2.8 .{ }^{16}$ The Cobra engineering model actuators have been assembled to a prototype module and tested. The results show satisfactory target convergence performance in the patrol field of each fiber. ${ }^{17}$ These subcomponents will be integrated into PFI and be fully tested at ASIAA before delivery to Subaru. ${ }^{18}$

Metrology Camera System (MCS) is under development at ASIAA. ${ }^{19}$ It will be installed at the Cassegrain focus of the telescope. Because the fiber positioners have no encoders, an external system is required to drive them to the proper position. MCS corresponds to this external system which takes images of the science and fiducial fibers back-lit from the other side of prime focus, and then measures the fiber positions, enabling closedloop operation of the positioners. MCS is capable of taking an image of all the back-lit science and fiducial fibers on the prime focus in one exposure. The fiber configuration time is significantly shorter than FMOS for which a small CCD camera needs to scan the field of view to measure all the fibers. The $380 \mathrm{~mm}$ aperture system is designed to minimize the impacts of the dome seeing effect and small-scale figure errors of the WFC lens surface shapes.

Spectrograph System (SpS) will be integrated at LAM ${ }^{\boldsymbol{N},}{ }^{20}$ with the fiber system delivered by LNA and the camera dewars \& detectors developed by Princeton University (PU) ${ }^{21}$ and Johns Hopkins University (JHU). ${ }^{22,23}$ The divergent beams from the science fibers on the pseudo slit are collimated and then split into blue, red and NIR channels by two dichroic mirrors. After this, the beam is dispersed by the VPH grating and spectral images are formed on the detectors. A grating exchange mechanism allows a higher dispersion VPH grism to be accommodated in the system and deliver medium resolution spectra in the red channel with no changes in the other parts of SpS. SpS consists of four spectrograph modules (SM) each of which is identically designed to deliver $\sim 600$ spectral images on the detectors.

Fiber system "FOCCoS" to be delivered by LNA ${ }^{24}$ consists of three parts: Two short-fiber systems accommodated in PFI and SpS, and a long cable system between them routed on the telescope. The route of this long one is still being finalized, but the total fiber length will be approximately $65 \mathrm{~m}$. These three parts are connected together by two sets of fiber connectors. One is needed at the telescope top end to make POpt2 detachable from the telescope, and the other is in front of SpS to ease the delivery and integration of SpS at Subaru and to make the operation and maintenance activities independent of the other PFS subsystems.

In Table 1, the major instrument parameters are listed. While the basic parameters are fixed, one should refer to the PFS official web site http://pfs.ipmu.jp/ for the fiber reconfiguration time, throughput, estimated on-sky sensitivity and related details as they will be updated as the instrument is built, integrated and tested and its characteristics are better understood.

\section{OPERATION CONCEPT}

For the operation of the PFS instrument and the large survey program it will carry out, coordination not only between the hardware and software but also between different software packages is crucial. Four software components are under development for this, with different sets of functions packaged and therefore designed to

\footnotetext{
${ }^{*}$ California Institute of Technology

${ }^{\dagger}$ Jet Propulsion Laboratory

${ }^{\ddagger}$ Laboratório Nacional de Astrofísica (Brazil)

$\S$ Academia Sinica Institute of Astronomy and Astrophysics (Taiwan)

`Laboratoire d'Astrophysique de Marseille
} 
Table 1. PFS major instrument parameters

\begin{tabular}{|c|c|c|c|c|}
\hline \multicolumn{5}{|c|}{ Prime Focus Instrument (PFI) } \\
\hline Field of view (hexagonal) & \multicolumn{4}{|c|}{$\begin{array}{c}\text { Diameter of circumscribed circle: } 1.38 \mathrm{deg} \\
\text { Area: } 1.25 \mathrm{deg}^{2}\end{array}$} \\
\hline Number of fibers & \multicolumn{4}{|c|}{2394 science fibers and 96 fixed fiducial fibers. } \\
\hline Fiber density & \multicolumn{4}{|c|}{$2000 \operatorname{deg}^{-2}\left(0.6 \operatorname{arcmin}^{-2}\right)$} \\
\hline Fiber core diameter & \multicolumn{4}{|c|}{$127 \mu \mathrm{m}(=1.12(1.02)$ arcsec at the field center (edge), respectively) } \\
\hline Positioner pitch & \multicolumn{4}{|c|}{$8 \mathrm{~mm}(=90.4(82.4)$ arcsec at the field center (edge), respectively) } \\
\hline Positioner patrol field diameter & \multicolumn{4}{|c|}{$9.5 \mathrm{~mm}(=107.4(97.9)$ arcsec at the field center (edge), respectively) } \\
\hline Fiber minimum separation & \multicolumn{4}{|c|}{$\sim 30$ arcsec } \\
\hline Fiber configuration time & \multicolumn{4}{|c|}{$\sim 60-100 \mathrm{sec}(\mathrm{TBC})$} \\
\hline Number of AG cameras & \multicolumn{4}{|c|}{6} \\
\hline Field of view per AG camera & \multicolumn{4}{|c|}{$5.1 \operatorname{arcmin}^{2}$} \\
\hline Sensitivity of AG camera & \multicolumn{4}{|c|}{$S / N=30(100)$ for $r=20 \mathrm{mag}(\mathrm{AB}), 1(10)$ sec exposure. } \\
\hline \multicolumn{5}{|c|}{ Spectrograph System (SpS) } \\
\hline \multirow{2}{*}{ Spectral arms } & \multirow{2}{*}{ Blue } & & & \multirow{2}{*}{ NIR } \\
\hline & & Low Res. & Mid. Res. & \\
\hline Spectral coverage & $380-650 \mathrm{~nm}$ & 630-970nm & $710-885 \mathrm{~nm}$ & $940-1260 \mathrm{~nm}$ \\
\hline Dispersion & $0.7 \AA /$ pix & $0.9 \AA / \mathrm{pix}$ & $0.4 \AA / \mathrm{pix}$ & $0.8 \AA / \mathrm{pix}$ \\
\hline Spectral resolution & $2.1 \AA$ & $2.7 \AA$ & $1.6 \AA$ & $2.4 \AA$ \\
\hline Resolving power & 2300 & 3000 & 5000 & 4300 \\
\hline SpS throughput & $53 \%($ at $500 \mathrm{~nm})$ & $57 \%$ (at $800 \mathrm{~nm})$ & $54 \%$ (at $800 \mathrm{~nm})$ & $33 \%$ (at $1100 \mathrm{~nm})$ \\
\hline
\end{tabular}

be only loosely coupled to each other. The detailed definitions of these four packages have been evolving as the instrument and survey operation concepts are being updated to maximally accommodate the distinct features of the planned survey for PFS SSP such as: (1) A much fainter limit than previous legacy surveys such as SDSS is pursued, exploiting the large light-gathering power of the Subaru Telescope, (2) given the wide variety of scientific goals, a wide variety of objects are targeted for observation and therefore a variety of definitions of success need to be encompassed, (3) PFS allows dynamic fiber reallocation even on an individual exposure basis, unlike the static integration in case of classical multi-slit and multi-fiber spectroscopy using machined plates. In addition, since PFS will be a facility instrument at the Subaru Telescope observatory and will be operated in the framework of general open-use observation, we are continually discussing all aspects of operations with the observatory and are trying to adapt our plans accordingly. Below an overview is given of the current operation concepts and the main bodies of the software definitions. Technical details are covered in another article. ${ }^{25}$

\subsection{Observation preparation}

The observation process starts with preparing an input target catalog which includes not only science targets but also stars for field acquisition, auto-guiding \& focusing, and flux calibration. Then telescope pointings, position angles, and fiber allocations to science targets at each pointing are defined for a given time of observation. This planning task is performed by a software package called "ETS" (Exposure Targeting Software) being developed by MPA". This observation configuration is prepared by scientists at sites off the observatory, and the prepared configuration is then uploaded to a database system. At the actual time of observation, which may be different from the one in the plan, the details in the mapping between the science targets and allocated fibers are recalculated before the fiber configuration starts. Observers should assign a fraction of fibers to observe blank sky regions and another fraction to observe flux calibration stars simultaneously with the science targets. The optimal numbers and distributions of these fibers for sky and calibration targets will be determined in the course of on-sky engineering observations.

\footnotetext{
" Max-Planck-Institut für Astrophysik
} 


\subsection{Instrument operation \& data acquisition}

"ICS" (Instrument Control Software) is the software package that orchestrates the PFS subsystems and subcomponents for instrument operation in coordination with the telescope system. The key component for the integration and coordination is a messaging hub system (MHS). As has been demonstrated in the SDSS operations at Apache Point Observatory, it efficiently organizes distributed processes providing uniform communication interfaces between subcomponents. MHS is being used for the operation of the CHARIS instrument ${ }^{26}$ (a high contrast integral field spectrograph for studying disks and extrasolar planets around stars) which is now in the commissioning phase at the Subaru Telescope, so we will take advantages of this experience in advance of the PFS commissioning process.

In actual observations, the first step is to point the telescope to the target field and set the instrument rotator to a requested angle. In parallel, the Hexapod, Atmospheric Dispersion Corrector, and telescope primary mirror active support are adjusted by the telescope control system for this new field. Once the telescope and rotator stop slewing and start operating in the tracking mode, exposures of acquisition stars are taken by the A\&G cameras, errors in the telescope pointing and rotator angle are calculated, and feedback is sent to the telescope control system for corrections. This process is iterated continuously until the errors are small enough to start auto-guiding. A focusing operation can be executed at some point in this course of field acquisition and auto-guiding.

While the telescope is slewing, the fiber positioner system can start configuring the science fibers at least coarsely to the expected positions of the science targets on the focal plane. The MCS measures the current positions of the science fibers and errors from the requested positions. Based on this information the fiber positions are updated and the errors get smaller as successive iterations are applied. Once the telescope and rotator are in the tracking mode, the rotator operation and auto-guiding are temporarily stopped ${ }^{* *}$ for fine positioning of the fibers (the telescope can still be moving in the tracking mode). Apart from the time for telescope slewing and rotator operation, one iteration of fiber configuration is expected to take $\sim 10-15 \mathrm{sec}$, including both the time of Cobra moves and exposure time of MCS. We expect $\sim 6$ iterations will be required, so one fiber configuration will be completed in $\sim 90 \mathrm{sec}$ given several iterations are needed, but more studies are ongoing to fully understand the timing budget.

Once the fiber configuration is complete, the rotator operation and the auto-guiding operation are restarted, and the spectrograph system then starts taking exposures as requested. The data format from each exposure are two $4 \mathrm{~K} \times 2 \mathrm{~K}$ CCDs from the blue and red cameras, and one $4 \mathrm{~K} \times 4 \mathrm{~K}$ H4RG detector from the NIR camera. At the end of each exposure, the data are read out and passed on to the Data Reduction Pipeline (DRP) for on-site data reduction, data quality assessment \& assurance, and data archival.

Below are a few details to be highlighted in the procedure of observation and data acquisition procedure:

- It is not trivial to determine that the Cobras are aligned well enough to the target positions. Actual criteria for convergence and procedure of assessment are still under discussions, but we consider that the concept of a signal-to-noise ratio can be useful as a measure of optimality, rather than the residual distances of the fibers from their targets. Starting from a situation in which all the fibers are remote from their target positions (except for chance coincidence), in the first few iterations, all the fibers get closer to the targets, so the signal in terms of fluxes from the objects to the fibers increases quickly. However, the gain of the signal after each move of the Cobras gets smaller at later iterations because most of the fibers are already reasonably close to the targets, and then the gain becomes less than the loss due to the loss of the observing time for integration on the detectors. Therefore the number of iterations giving the optimal signal-to-noise ratio must be somewhere in the middle (Fig. 4). In reality, since the time for one iteration is rather short in particular at later iterations, some more iterations to pursue better positions of more fibers may be worth at relatively small expenses of observing time, so we will leave such flexibility in the choice.

${ }^{* *}$ When observations are carried out using the prime focus, the prime focus instrument can be rotated, but the Cassegrain instrument cannot be operated because the focus is not in use. Accordingly, the MCS cannot be rotated synchronously with PFI and therefore the images of back-illuminated fibers on MCS are elongated if PFI is rotated and could worsen the centroiding error. 

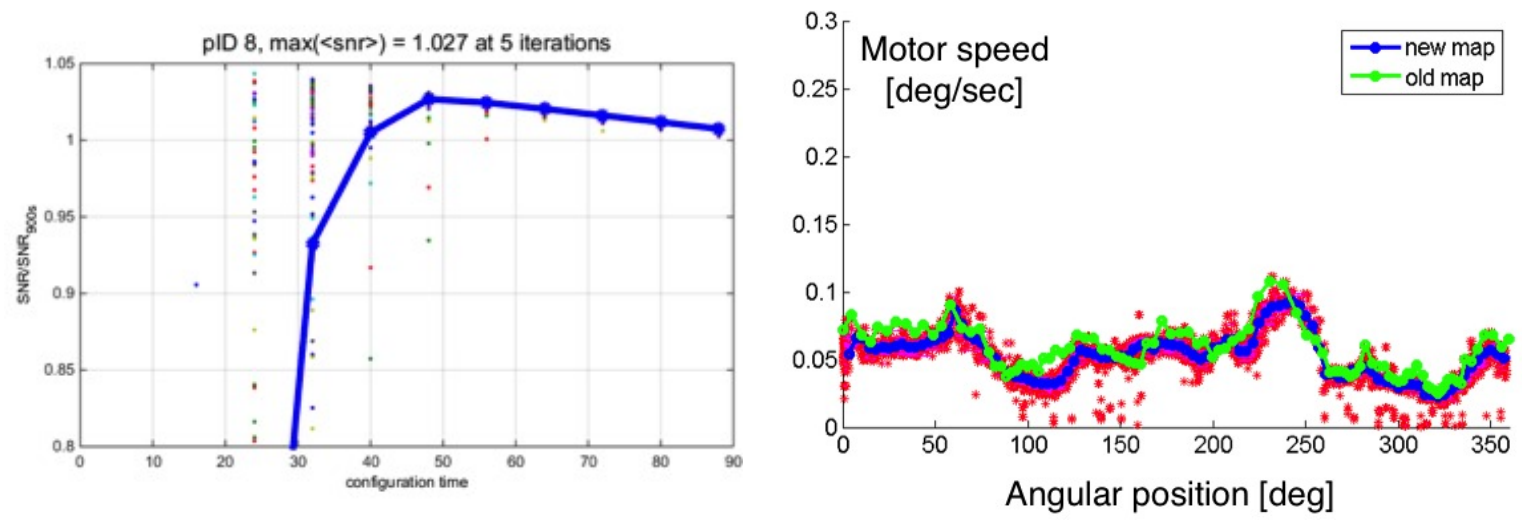

Figure 4. On the left, the level of convergence of Cobras to requested positions is plotted against measure of the signalto-noise ratio relative to a fiducial value, which increases to the maximum at the fourth iterations and slowly decreases at later iterations. These data were taken at the target convergence tests on the engineering model Cobra positioners module. On the right, the data points indicate the Cobra motor speeds as a function of angular position. The relationship is so-called a motor map. As two curves are shown in the graph, a motor map can be updated as more data are collected.

- The response of each motor in the Cobra actuator to a drive signal is known to depend on angular position (Fig. 4). Characterizing this so-called "motor map" and operating the Cobras taking it into account are considered key to achieve efficient convergence.

- Although observation strategies and data quality success criteria are different among the three main science areas, all three are planning to acquire data with no beam-switching to blank sky. (The instrument control system will accommodate the beam switching capability as an option.) This will maximize the on-source integration time over the observing time and minimize the geometric constraints in the allocation of science fibers to science targets ${ }^{\dagger \dagger}$.

- Due to the large field of view, the differential atmospheric refraction effect is severe over most of the sky. Accordingly, the science fibers need to be reconfigured e.g. every $\sim 30$ minutes (TBC).

- The instrument rotator operates over a restricted range between $-60 \mathrm{deg}$ to $+60 \mathrm{deg}$. This is to minimize any variation of the fiber status by instrument rotation (possibly important for stable Point Spread Function (PSF) on the spectrograph detectors and therefore for good sky subtraction), exploiting the hexagonal symmetry on the PFS focal plane.

\subsection{Data reduction \& spectral calibration strategy}

"DRP" (Data Reduction Pipeline) comprises the "2D" part (2D-DRP) and the "1D" part (1D-DRP). The 2D-DRP, which is under development by PU, receives two-dimensional raw spectral FITS images read out from the detectors and produces one-dimensional, sky-subtracted, flux- and wavelength-calibrated spectra ready for scientific analyses. The 1D-DRP being developed by LAM then receives these $1 \mathrm{D}$ spectra and measures various parameters of spectroscopic features such as redshifts and emission line fluxes. After each exposure of the spectrograph detectors, the data will be processed by on-site DRP with calibration data sets taken in advance. 1D-DRP is applied to the reduced and calibrated 1D spectra and measured parameters are added to the database. As successive exposures are taken for the same objects at different nights and observation runs, deeper and higher quality spectra will be produced from from full, batch processing of all available data.

One important challenge for this project is to achieve the goal of sky subtraction accuracy (down to $\sim 0.5 \%$ in the faint sky continuum between the lines). This means, given that we are not doing beam-switching operations,

\footnotetext{
${ }^{\dagger \dagger}$ In cross-beam switching observations, two fibers are assigned to one science target and the telescope pointing is dithered between one exposure and another so that in the first exposure one of the fibers is placed on the target and the other observes blank sky, and in the next exposure, they switch the role. This way, 100\% of the exposure time can be used for on-source integration, but the fibers can be significantly less flexibly allocated to targets.
} 
that the sky spectrum of an object fiber needs to be accurately modeled from the spectra of other fibers looking at the sky, and for this, the two-dimensional fiber PSF needs to be well characterized as functions of $\mathrm{x}$ and $\mathrm{y}$ on the detectors (corresponding to fibers and wavelengths approximately). In other words, the conditions during the calibration data acquisition need to closely mimic the observing conditions at night. We have the following plans for this:

- We have a calibration lamp system on top of PFI for both flat-fielding and wavelength calibration. This lamp illuminates a quasi-Lambertian (TBC) screen on the ceiling of the telescope enclosure and the illumination reflects back to the telescope primary mirror. In this way, the telescope pupil can be diffusely illuminated for calibration mimicking the illumination by the sky in real observations at night.

- Even if the pupil illumination is managed as above, differences of the fiber status between observations and calibration may cause some errors in the PSF modeling due to e.g. variation of Focal Ratio Degradation (FRD) in the fibers. There are three cases where such errors may be introduced: (1) Fiber moves (mainly twists) as the Cobras move, (2) coils/uncoils of fiber bundles with the rotation of the instrument by the rotator, and (3) bending/unbending of the fiber cable due to telescope elevation changes. We currently think that (1) has the most significant impact, so the procedure is likely to take the calibration data for every fiber configuration taken in a given night. Detailed studies are underway. If (2) is also significant, the rotator angle should also be reproduced in this data acquisition but the amount of calibration data required could be huge and it may not be realistic to take them all during a night. If (2) and/or (3) are significant, we will plan to have another calibration lamp system to take data in the daytime as functions of telescope elevation and rotator angle and characterize the impacts.

- A significant number of fibers should be assigned as sky fibers and should be roughly uniformly distributed over the focal plane. The required number is still TBD, and will be clarified in commissioning observations.

\subsection{Data quality assessment and assurance for long-term survey processing}

The procedure of data quality assessment and assurance (QA) is still being actively discussed in detail, but we are aiming to accommodate a data QA on an object-by-object basis: Data quality assessment procedure and success criteria are set for each object so that, once a particular object is considered "done", the fiber(s) assigned to the object can be allocated to a different target and be reconfigured accordingly. Also, discussions of a data QA in a short time scale (much shorter than one night) are underway, for which "on-site" (i.e. at the telescope) quick data reduction is needed in addition to "off-site" full reduction. In particular for faint objects, one of the key processes is full analysis of sky fibers even in the quick on-site data QA to understand the noise characteristics and subsequently limiting fluxes as a function of wavelength. The database is then updated with such information, revisions are applied to the field definitions and/or fiber configurations accordingly, and observations are performed using such updated fields and fiber configurations. This routine is repeated until the survey is considered "done". "SPT" (Survey Planning and Tracking software) is the software package for the survey management responsible for data QA.

\section{RECENT DEVELOPMENTS}

\subsection{The collaboration}

In December 2015, a consortium of Chinese institutes ${ }^{\ddagger \ddagger}$ including 11 senior scientists by Yipeng Jing (Shanghai) joined the PFS collaboration as a full member. To make the collaboration even stronger and to further improve the chance of success of instrument development and survey science, we are still looking for new partners. There are a few groups and institutes as candidates with which the PFS steering committee is negotiating. 


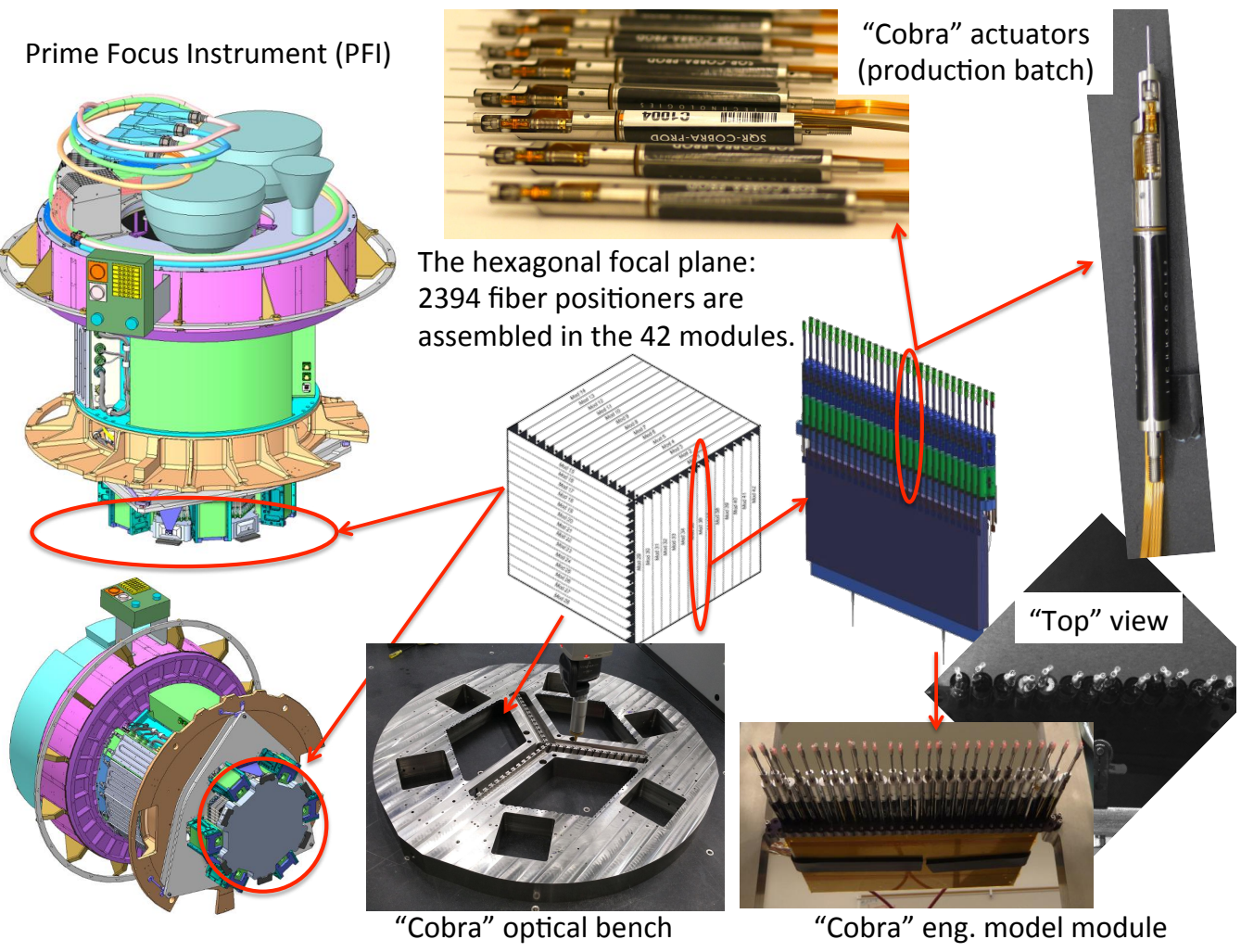

Figure 5. A schematic view of the PFS focal plane to be equipped with the 2394 fibers and Cobra actuators. Several photos of the real hardware components are also shown: The production-batch Cobra actuators (top right), a Cobra module with the engineering model actuators populated (bottom right), and the Cobra optical bench in process which is to accommodate 42 Cobra modules.

\subsection{The instrument development}

After the project passed the conceptual design review in March 2012 and the preliminary design review in February 2013, we also have gone through critical design reviews of most of the subsystems and subcomponents: Spectrograph system in March 2014, PFI in March 2015, fiber positioner system in June 2015, and metrology camera in September 2015, with additional delta reviews later when we considered they were needed. Subsequently, the construction, integration and test of the instrument are actively under way at the subsystem level. Obviously, there are quite a few challenges even in the procurement and production of individual components. Here, a few examples are briefly introduced, while updates in the other subsystems and subcomponents are presented in other articles by the PFS team: ${ }^{18-23}$

- The fiber positioner "Cobra" (Fig. 5): After several years of prototyping and testing activities, ${ }^{17}$ we recently started the mass production of the Cobras at New Scale Technologies. About 500 units have already been delivered as of Jun 2016. We are extracting two units approximately every month from the production batch and are sending them to the life test, where a specific cycle of motor moves is repeated until 400k times (c.f. we expect about $100 \mathrm{k}$ cycles to be accumulated in $\sim 10$ years operations of individual Cobras including those in engineering observations and calibration exposures) and the motor torques are measured at every $100 \mathrm{k}$ cycle. While one failure mode due to a rare manufacturing flaw of one specific

${ }_{\ddagger}$ The members are: Shanghai Jiao Tong University, Shanghai Astronomical Observatory, University of Science and Technology of China, Tsinghua University, Xiamen University, and National Astronomical Observatory of China. 


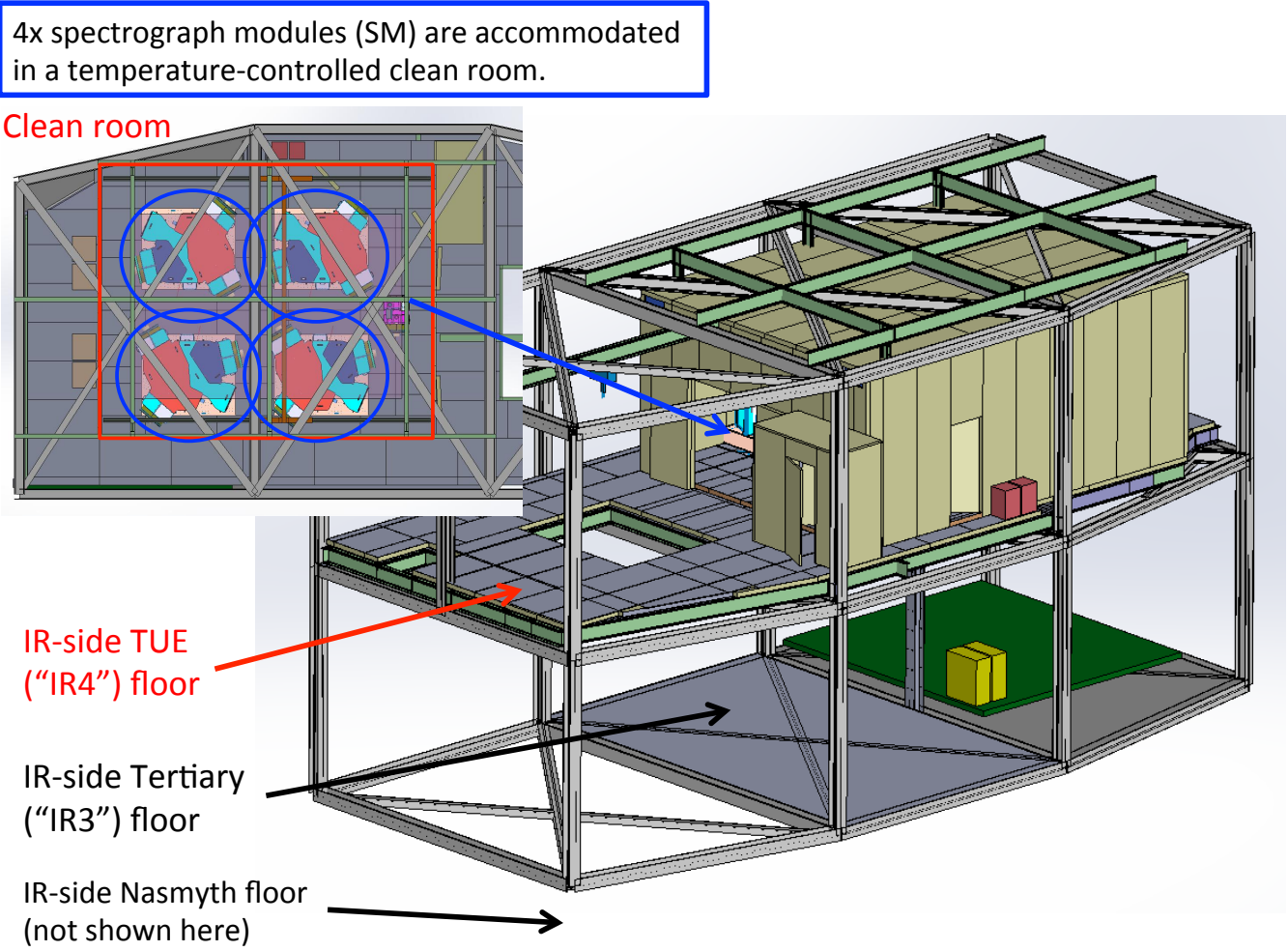

Figure 6. An overview based on the 3D model of the floor (IR4 floor) and temperature-controlled clean (SCR) where to accommodate the PFS spectrograph system.
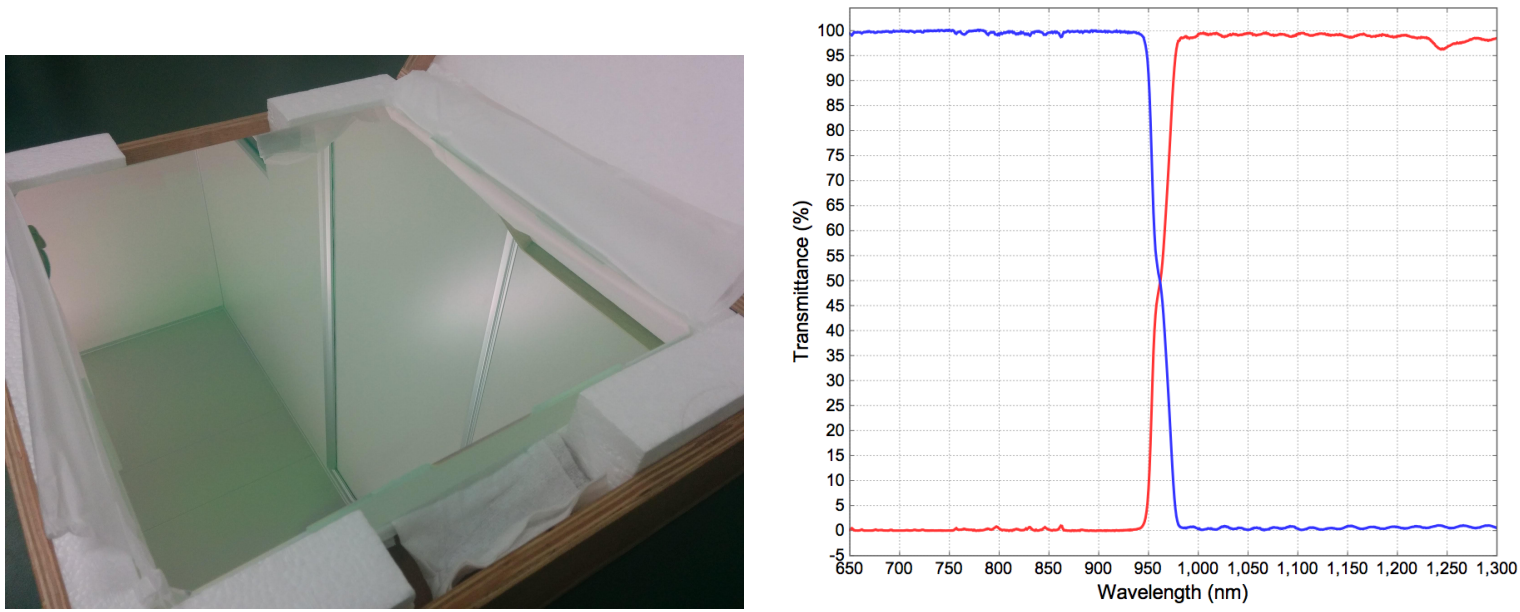

Figure 7. On the left, a photo of the first red dichroic mirror is shown. On the right, the measured reflectance (transmittance) is plotted as a function of wavelength by a blue (red) curve, respectively. The substrate was coated by Asahi Spectra Co., Ltd.. 

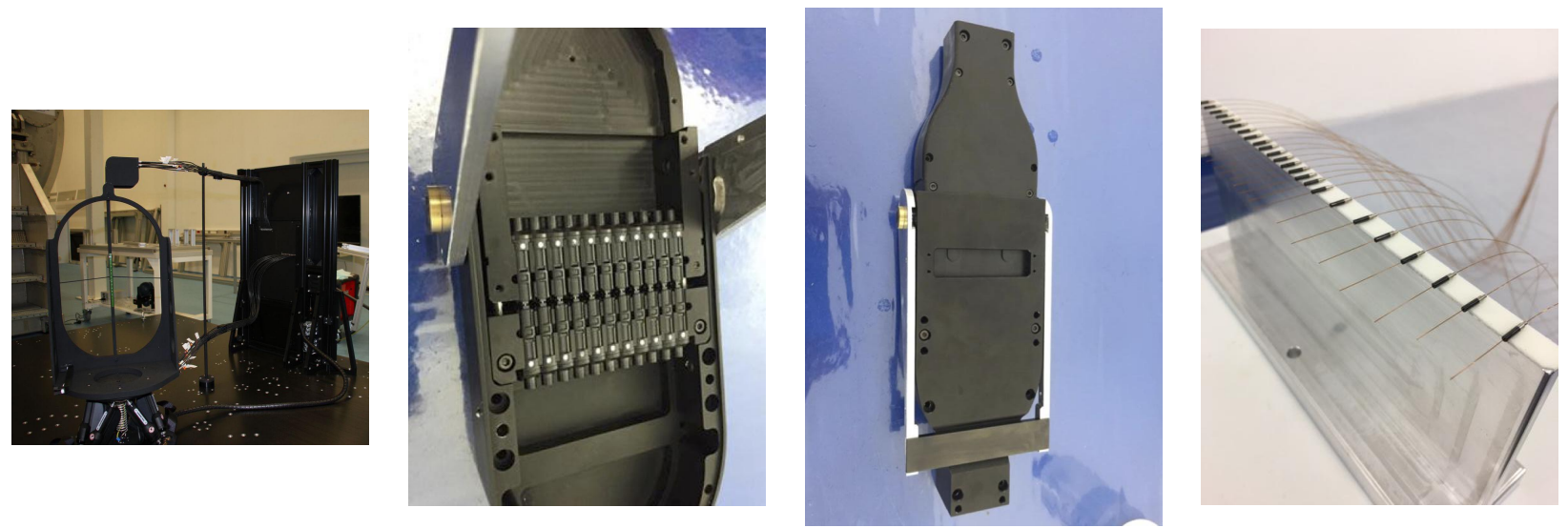

Figure 8. Photos of the parts and assemblies related to the fiber system: ${ }^{24}$ (Left) The SpS-side fiber cable system, (middle-left) the inside of the large-format custom-made fiber connector assembly ("Tower connector") to be installed on the telescope spider structure, (middle-right) the "Tower connector" when mated, and (right) the fibers and ferrules for the PFI-side fiber system in the integration process.

part was found in these sampled life tests and corrective actions were taken, the tests are successfully progressing and statistical evidence for durability is growing.

- The detailed design of SpS platform at the Subaru Telescope observatory: The four SMs will be accommodated in a temperature controlled clean room (Spectrograph Clean Room: SCR) on the "infrared-side" 4th floor of the telescope enclosure building ("IR4" floor). On this floor, the spectrograph systems of FMOS were operated, but the existing floor is considered inappropriate to accommodate PFS SpS due mainly to the significantly higher weight and different distribution. In 2015, design studies of the IR4 floor mechanical structure and SCR are intensively performed by T. Tamura (Subaru) (Fig. 6). The FMOS spectrograph systems are now being dismantled and removed from the floor for the restructuring works to be started. Currently detailed scheduling, logistics and coordination after the floor is restructured and SCR is prepared are under discussions between the observatory and PFS team.

- The dichroic coating to split the collimated beam in the spectrograph into the blue, red, and NIR channels: The difficulty is to meet the requirement of the sharp transition $(\sim 20 \mathrm{~nm})$ between the reflection and transmission as well as the high throughput in each regime ( $\geq 95 \%$ of reflectance and $\geq 90 \%$ of transmittance), but products with satisfactory properties have started being delivered (Fig. 7).

- The fiber system: Although the "telescope part" of the fiber cable system connecting PFI and SpS is still being prototyped, the integration and test are under way for the other parts which connect the PFI and SpS (Fig. 8). The procedure for implementing optimal quality control is being finalized.

\subsection{Instrument characterization}

In parallel to these construction, integration and test activities for the subsystems, the detailed plans of the commissioning process are under development (Fig. 9). The commissioning is divided in two parts: the reintegration and test of the subsystems at the observatory and engineering observations on the telescope. The plan needs to be elaborated by discussions with the observatory for detailed configurations with various resources and constraints at the observatory. It should also be optimized in terms of efficiency making sure that we will take full advantage of the complementarity with the advanced subsystem-level integration and test processes. We are now listing the tasks that can be completed off the telescope and those to be done on the telescope, coordinate them along a time sequence according to the dependencies between them, and develop a schedule of engineering observations which will likely be separated into several runs each of which will span a few to several nights. One of the important works at the beginning of engineering observations is to understand the way of operating the Hexapod of POpt2 for the alignment of PFI with WFC, initially by using the AG cameras only. 


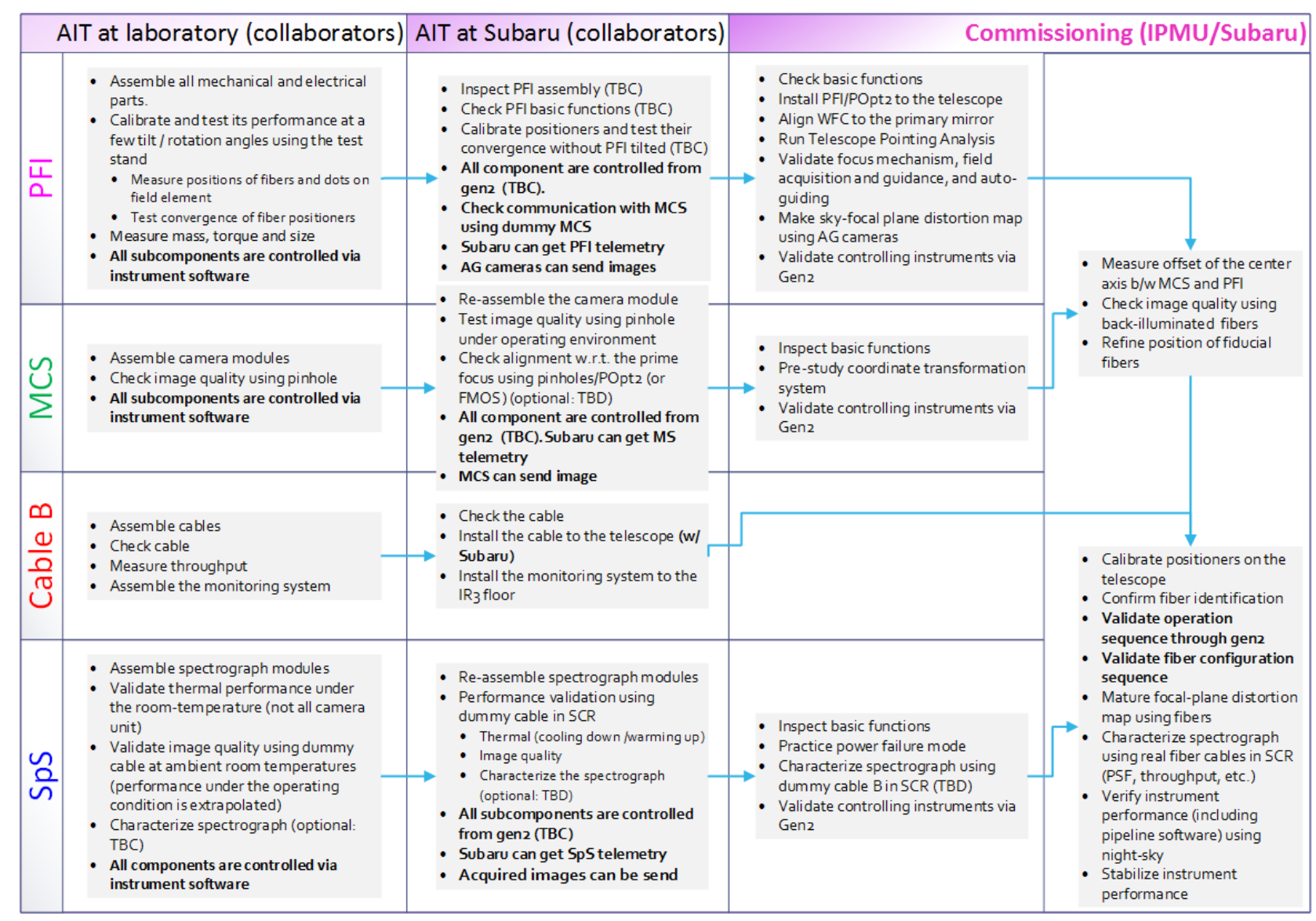

Figure 9. One representation of the commissioning process, highlighting the transition from the subsystem AIT (Assembly, Integration and Test) on the left, to engineering observations on the right. As the commissioning progresses, the work moves from the collaborators to the PFS project office and the observatory. As the subsystems are integrated into the system, the tasks for system-level operation and performance validation increase.

Y. Tanaka (Subaru) defined efficient diagnostics of tilt and de-center and is developing a routine of taking AG camera images of bright stars and applying corrections so that the alignment can be achieved in a few iterations. In the next couple of years, we will try to adaptively mature the plan as the subsystem integration and test at the subsystem level progress.

For the studies of on-sky sensitivity and survey planning, a spectrum simulator has been prepared by C. Hirata (Ohio State), K. Yabe (Kavli IPMU), and R. Lupton (Princeton), modifying the exposure time calculator for the WFIRST project ${ }^{27}$ with the relevant set of information for PFS including an updated throughput model. The output from this simulator has been matched with the preliminary data model for PFS that has recently been defined based on that of SDSS, and it will be used to develop and validate the software components such as 1D-DRP and the database. Meanwhile, the Princeton team has been developing a simulator of raw images from the spectrograph detectors where the ray-trace calculations of the optical model and detector characteristics such as bias and dark are considered. These simulated 2D images (Fig. 10) and spectra have been used to develop and validate the $2 \mathrm{D}$-DRP. They have coded a prototype pipeline, with which the data from the real spectrograph module will be reduced and analyzed during the integration and test at LAM. The data will be crucial not only to characterize the spectrograph module but also to develop the algorithms of 2D-DRP for data modeling such as PSF characterization. 

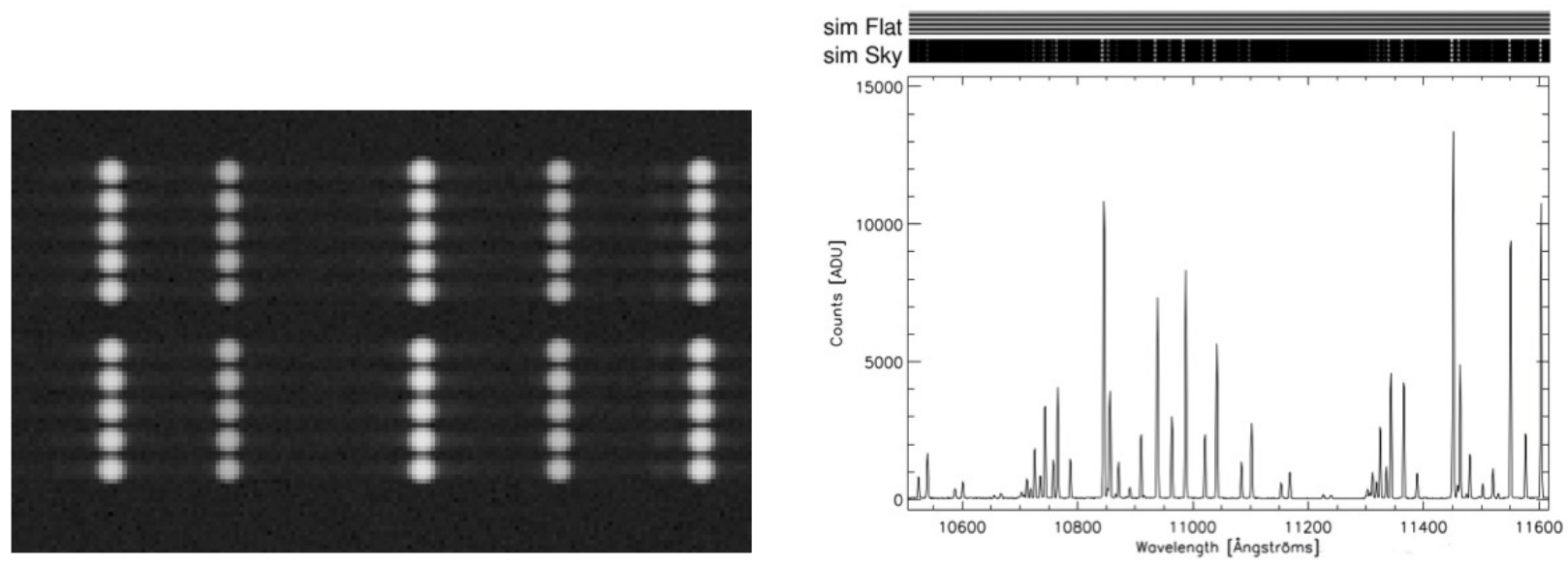

Figure 10. Examples of simulated images and spectra for the 2D-DRP development: a few Neon and Argon lines on the left, and $\mathrm{OH}$ night-sky lines on the right.

\section{SUMMARY AND FUTURE PERSPECTIVES}

PFS (Prime Focus Spectrograph), a next generation facility instrument on the Subaru Telescope, is a very wide-field, massively multiplexed, optical and NIR spectrograph: The prime focus will be equipped with 2394 reconfigurable fibers in the $1.3 \mathrm{deg}$ field of view, and the spectra simultaneously cover the wide range of wavelengths from $380 \mathrm{~nm}$ to $1260 \mathrm{~nm}$ at one exposure. The development of this instrument by an international collaboration under the initiative of Kavli IPMU is finalizing the design and starting the construction at the subsystem level. We expect the subsystems to be integrated and validated by the collaborators and be delivered to the telescope site in 2017-2018. We will then carry out system integration and start engineering observations from early 2018. Based on our preliminary plans, $\sim 1.5$ years will be necessary to complete engineering observations (including a certain period for optimization and stabilization of the performance and operation), so we expect to start science operation and a 5-year PFS SSP survey from mid-late 2019. The science team is developing a detailed survey strategy to be refined in the next two years, and the technical team is committing to this by brushing up the estimates of on-sky instrument sensitivity and carrying out survey and data simulations. Information on the instrument development and survey strategy will be posted and updated on the PFS official website http://pfs.ipmu.jp/. In addition, news, events and milestones are reported in the PFS official blog http://pfs.ipmu.jp/blog/.

PFS and HSC, a unique set of powerful survey instruments, will be crucial strategic pieces for the Subaru Telescope through the 2020s into the 2030s, allowing unique science by effective synergies with new generation ground-based and space missions such as TMT, LSST, Euclid and WFIRST.

\section{ACKNOWLEDGMENTS}

We appreciate all the contributions from the PFS science team to the instrument requirements definitions and the survey planning. We also thank the people involved with this PFS project in the past in any formats. Without their efforts and contributions, the project would not even exist now. We are grateful to the staffs (in addition to those on the author list) at National Astronomical Observatory of Japan and the Subaru Telescope observatory for their contributions to the development of the PFS instrument, the modifications of the telescope system and other infrastructures to accept PFS, the preparations of PFS system integration and engineering observations, and various other aspects such as the administrative supports. Our thanks should also go to the staffs at Durham University, UK, for their supports of the development of the PFS fiber system as the consultancy. We are grateful to the external reviewers at the PFI critical design review (Kim Aaron, Mark Colavita, Randy Foehner, Kirk Seaman from JPL, French Leger from University of Washington, and Ted Huang from ASIAA) for their insightful and valuable inputs. We gratefully acknowledge support from the Funding Program for World-Leading Innovative R\&D on Science and Technology (FIRST) program "Subaru Measurements of Images and Redshifts (SuMIRe)", 
CSTP, Japan. This work is supported by JSPS KAKENHI Grant Numbers JP15H05893, JP15K21733, and JP15H05892. The work in ASIAA, Taiwan, is supported by the Academia Sinica of Taiwan. The work in Brazil is supported by the FAPESP grant 2012/00800-4.

\section{REFERENCES}

[1] Miyazaki, S., Komiyama, Y., Sekiguchi, M., Okamura, S., Doi, M., Furusawa, H., Hamabe, M., Imi, K., Kimura, M., Nakata, F., Okada, N., Ouchi, M., Shimasaku, K., Yagi, M., and Yasuda, N., "Subaru Prime Focus Camera - Suprime-Cam," PASJ 54, 833-853 (2002).

[2] Kimura, M., Maihara, T., Iwamuro, F., Akiyama, M., and Tamura, N. et al., "Fibre Multi-Object Spectrograph (FMOS) for the Subaru Telescope," PASJ 62, 1135-1147 (2010).

[3] Tamura, N., Takato, N., Iwamuro, F., Akiyama, M., and Kimura, M. et al., "Subaru FMOS now and future," in [Ground-based and Airborne Instrumentation for Astronomy IV], Ian S. McLean, Suzanne K. Ramsay, H. T., ed., Proc. SPIE 8446, 8446M0 (2012).

[4] Brzeski, J. K., Gillingham, P., Correll, D., Dawson, J., Moore, A. M., Muller, R., Smedley, S., and Smith, G. A., "Echidna: the engineering challenges," in [Ground-based Instrumentation for Astronomy], Moorwood, A. F. M. and Masanori, I., eds., Proc. SPIE 5492, 1228-1242 (2004).

[5] Yabe, K., Ohta, K., Iwamuro, F., Akiyama, M., and Tamura, N. et al., "The mass-metallicity relation at $z \sim 1.4$ revealed with Subaru/FMOS," MNRAS 437(4), 3647-3663 (2014).

[6] Mužić, K., Scholz, A., Geers, V., Jayawardhana, R., and Tamura, M., "Substellar Objects in Nearby Young Clusters (SONYC). V. New Brown Dwarfs in $\rho$ Ophiuchi," ApJ 744(2), 134, 11 pp. (2012).

[7] Tonegawa, M., Totani, T., Okada, H., Akiyama, M., and Dalton, G. B. et al., "The Subaru FMOS galaxy redshift survey (FastSound). I. Overview of the survey targeting h $\alpha$ emitters at $z \sim 1.4, "$ PASJ 67(5), id.8112 pp. (2015).

[8] Okumura, T., Hikage, C., Totani, T., Tonegawa, M., and Okada, H. et al., "The Subaru FMOS galaxy redshift survey (FastSound). IV. New constraint on gravity theory from redshift space distortions at $z \sim 1.4$," PASJ 68(3), id.3824 pp. (2016).

[9] Miyazaki, S., Komiyama, Y., Nakaya, H., Kamata, Y., and Doi, Y. et al., "Hyper Suprime Cam," in [Ground-based Instrumentation for Astronomy], Moorwood, A. F. M. and Masanori, I., eds., Proc. SPIE 5492, 1228-1242 (2012).

[10] Levi, M., Bebek, C., Beers, T., Blum, R., Cahn, R., Eisenstein, D., Flaugher, B., Honscheid, K., Kron, R., Lahav, O., McDonald, P., Roe, N., and Schlegel, D., "The DESI Experiment, a whitepaper for Snowmass 2013." arXiv:1308.0847.

[11] Spergel, D., Gehrels, N., Baltay, C., Bennett, D., Breckinridge, J., Donahue, M., Dressler, A., Gaudi, B. S., Greene, T., Guyon, O., Hirata, C., Kalirai, J., Kasdin, N. J., Macintosh, B., Moos, W., Perlmutter, S., Postman, M., Rauscher, B., Rhodes, J., Wang, Y., Weinberg, D., Benford, D., Hudson, M., Jeong, W.-S., Mellier, Y., Traub, W., Yamada, T., Capak, P., Colbert, J., Masters, D., Penny, M., Savransky, D., Stern, D., Zimmerman, N., Barry, R., Bartusek, L., Carpenter, K., Cheng, E., Content, D., Dekens, F., Demers, R., Grady, K., Jackson, C., Kuan, G., Kruk, J., Melton, M., Nemati, B., Parvin, B., Poberezhskiy, I., Peddie, C., Ruffa, J., Wallace, J. K., Whipple, A., Wollack, E., and Zhao, F., "Wide-Field InfrarRed Survey Telescope-Astrophysics Focused Telescope Assets WFIRST-AFTA 2015 Report." arXiv:1503.03757.

[12] Bouwens, R. J., Illingworth, G. D., Oesch, P. A., Stiavelli, M., van Dokkum, P., Trenti, M., Magee, D., Labb, I., Franx, M., Carollo, C. M., and Gonzalez, V., "Discovery of $z \sim 8$ Galaxies in the Hubble Ultra Deep Field from Ultra-Deep WFC3/IR Observations," ApJ Letters 709(2), L133-L137 (2010).

[13] Walker, M. G., Mateo, M., and Olszewski, E. W., "Stellar Velocities in the Carina, Fornax, Sculptor, and Sextans dSph Galaxies: Data From the Magellan/MMFS Survey," $A J$ 137(2), 3100-3108 (2009).

[14] McConnachie, A. W., "The Observed Properties of Dwarf Galaxies in and around the Local Group," $A J$ 144(1), article id. 4, 36 pp. (2012).

[15] Takada, M., Ellis, R. S., Chiba, M., Greene, J. E., and Aihara, H. et al., "Extragalactic science, cosmology, and galactic archaeology with the Subaru Prime Focus Spectrograph," PASJ 66(1), id.R1 (2014). 
[16] Takato, N., Tanaka, Y., Gunn, J. E., Tamura, N., and Shimono, A. et al., "Design and performance of a F/\#-conversion microlens for prime focus spectrograph at Subaru Telescope," in [Ground-based and Airborne Instrumentation for Astronomy V], Suzanne K. Ramsay, Ian S. McLean, H. T., ed., Proc. SPIE 9147, 914765 (2014).

[17] Fisher, C., Morantz, C., Braun, D., Seiffert, M., and Aghazarian, H. et al., "Developing engineering model cobra fiber positioners for the Subaru Telescope's prime focus spectrometer," in [Advances in Optical and Mechanical Technologies for Telescopes and Instrumentation], Ramón Navarro, Colin R. Cunningham, A. A. B., ed., Proc. SPIE 9151, 91511 Y (2014).

[18] Wang, S.-Y., Schwochert, M. A., Huang, P.-J., Chen, H.-Y., Kimura, M., Hu, Y.-S., Chou, C.-Y., Chang, Y.-C., Ling, H.-H., Hsu, S.-F., Morantz, C. N., Reiley, D. J., Mao, P. H., Braun, D. F., Wen, C.-Y., Yan, C.-H., Karr, J. E., Murray, G. J., Gunn, J. E., Tamura, N., Takato, N., and Shimono, A., "The current status of prime focus instrument of Subaru prime focus spectrograph," in [Ground-based and Airborne Instrumentation for Astronomy VI], Proc. SPIE 9908 (2016).

[19] Wang, S.-Y., Chou, C.-Y., Huang, P.-J., Ling, H.-H., Karr, J. E., Chang, Y.-C., Hu, Y.-S., Hsu, S.-F., Chen, H.-Y., Gunn, J. E., Reiley, D. J., Tamura, N., Takato, N., and Shimono, A., "Metrology camera system of prime focus spectrograph for Subaru telescope," in [Ground-based and Airborne Instrumentation for Astronomy VI], Proc. SPIE 9908 (2016).

[20] Madec, F., Le Mignant, D., Barrette, R., Belhadi, M., Blanchard, P., Dohlen, K., Ferrand, D., Jaquet, M., Le Fur, A., Le Merrer, J., Pascal, S., Gunn, J. E., Smee, S. A., Tamura, N., and Shimono, A., "SUBARU prime focus spectrograph: integration, testing and performance for the first spectrograph," in [Ground-based and Airborne Instrumentation for Astronomy VI], Proc. SPIE 9908 (2016).

[21] Gunn, J. E., Fitzgerald, R., Hart, M., Hope, S. C., Loomis, C., Peacock, G. O., Golebiowski, M., Carr, M., Smee, S. A., Tamura, N., Shimono, A., and Takato, N., "Detector and control system design and performance for the SuMIRe Prime Focus Spectrograph (PFS) cameras," in [Ground-based and Airborne Instrumentation for Astronomy VI], Proc. SPIE 9908 (2016).

[22] Smee, S. A., Gunn, J. E., Golebiowski, M., Hope, S. C., Madec, F., Gabriel, J.-F., Loomis, C., Le fur, A., Dohlen, K., Le Mignant, D., Barkhouser, R., Carr, M. A., Hart, M., Tamura, N., Shimono, A., and Takato, N., "Visible camera cryostat design and performance for the SuMIRe Prime Focus Spectrograph (PFS)," in [Ground-based and Airborne Instrumentation for Astronomy VI], Proc. SPIE 9908-333 (2016).

[23] Hart, M., Barkhouser, R. H., Smee, S. A., Gunn, J. E., and Loomis, C. P., "Detector characterization for the Subaru prime focus spectrograph (PFS)," in [Ground-based and Airborne Instrumentation for Astronomy VI], Proc. SPIE 9915 (2016).

[24] de Oliveira, A. C., de Oliveira, L. S., de Arruda, M. V., Souza Marrara, L., and dos Santos, L. H. et al., "Fiber optical cable and connector system (FOCCoS) for PFS/Subaru," in [Advances in Optical and Mechanical Technologies for Telescopes and Instrumentation], Ramón Navarro, Colin R. Cunningham, A. A. B., ed., Proc. SPIE 9151, 91514G (2014).

[25] Shimono, A., Tamura, N., Takato, N., Yasuda, N., Suzuki, N., Loomis, C., Lupton, R. H., Moritani, Y., and Yabe, K., "The survey operation software system development for Prime Focus Spectrograph (PFS) on Subaru Telescope," in [Software and Cyberinfrastructure for Astronomy IV], Proc. SPIE 9913 (2016).

[26] Groff, T. D., Kasdin, N. J., Galvin, M., Knapp, G. R., Carr, M. A., Brandt, T. D., Peters-Limbach, M. A., Loomis, C. P., Jarosik, N., Guyon, O., Jovanovic, N., McElwain, M. W., Takato, N., and Hayashi, M., "Laboratory testing and commissioning of the CHARIS integral field spectrograph," in [Ground-based and Airborne Instrumentation for Astronomy VI], Proc. SPIE 9908-23 (2016).

[27] Hirata, C. M., Gehrels, N., Kneib, J.-P., Kruk, J., Rhodes, J., Wang, Y., and Zoubian, J., "The WFIRST Galaxy Survey Exposure Time Calculator." arXiv:1204.5151. 